Original Article

\title{
Acute effects of accumulated aerobic exercise on aortic and peripheral pulse wave velocity in young males
}

\author{
Ryota Kobayashi, PhD ${ }^{1)^{*}}$, Hiroyuki Hatakeyama ${ }^{2)}$, Yuto Hashimoto ${ }^{2)}$, \\ TAKANOBU OKамото, $\mathrm{PhD}^{3)}$ \\ 1) Center for Fundamental Education, Teikyo University of Science: 2-2-1 Senju, Sakuragi, Adachi- ku, \\ Tokyo 120-0045, Japan \\ 2) Graduate School of Health and Sport Science, Nippon Sport Science University, Japan \\ 3) Department of Exercise Physiology, Nippon Sport Science University, Japan
}

\begin{abstract}
Purpose] The present study investigates the acute effects of accumulated aerobic exercise on aortic and peripheral pulse wave velocity (PWV) in healthy young males. [Subjects and Methods] We gathered data from 9 healthy young males (age, $23.4 \pm 0.6$ years) performed aerobic exercise group (AE, $65 \%$ peak oxygen uptake; two 15 min bouts of cycling performed with 20 min rest) and control group (CON, seated and resting in a quiet room) on separate days in a randomized, controlled crossover fashion. Carotid-femoral (aortic) and femoral-ankle (peripheral) PWV, carotid augmentation index, carotid, brachial and ankle blood pressure and heart rate were measured before (baseline) and 20 min for both groups. [Results] Aortic and peripheral PWV decreased from baseline at 20 min for the AE group, but not in the CON group. [Conclusion] The present findings indicate that acute accumulated aerobic exercise decreases aortic and peripheral PWV in healthy young males.

Key words: Accumulated aerobic exercise, Aortic and peripheral pulse wave velocity, Carotid augmentation index
\end{abstract}

(This article was submitted Sep. 27, 2017, and was accepted Oct. 30, 2017)

\section{INTRODUCTION}

Increased aortic and peripheral pulse wave velocity (PWV) are indices of arterial stiffness and are major risk factors for cardiovascular disease ${ }^{1,2)}$. Aortic PWV influences cardiovascular disease to a greater extent than does peripheral PWV ${ }^{3)}$. Therefore, to suppress the onset of cardiovascular disease, it is important to suppress increases in aortic PWV.

Acute continuous aerobic exercise decreases $\mathrm{PWV}^{4}$. Heffernan et al. ${ }^{5)}$ showed that moderate-intensity $[65 \%$ peak oxygen uptake $\left.\left(\mathrm{VO}_{2 \text { peak }}\right)\right]$ acute continuous aerobic exercise for 30 min decreases aortic PWV after 20 min in healthy young males. Recently, it was shown that accumulated aerobic exercise decreases the cardio-ankle vascular index (CAVI), an index of systemic arterial stiffness ${ }^{6,7)}$. Zheng et al ${ }^{8)}$ reported that CAVI decreases after moderately intense acute accumulated aerobic exercise in healthy young males. However, the acute effects of accumulated aerobic exercise on aortic and peripheral PWV is not yet fully understood. The purpose of present study is to determine the acute effects of accumulated aerobic exercise on changes in aortic and peripheral PWV.

\section{SUBJECTS AND METHODS}

We gathered data from nine healthy young males (age $23.4 \pm 0.6$ years; height $172.5 \pm 2.3 \mathrm{~cm}$; weight $64.1 \pm 2.4 \mathrm{~kg}$, Table 1 ). All lived sedentary lifestyles, were normotensive, did not smoke, and had no symptoms or history of chronic diseases.

*Corresponding author. Ryota Kobayashi (E-mail: rkobayashi@ntu.ac.jp)

(C2018 The Society of Physical Therapy Science. Published by IPEC Inc. 
Table 1. Subject characteristics $(\mathrm{n}=9)$

\begin{tabular}{lc}
\hline & Value \\
\hline Age $($ years $)$ & $23.0 \pm 2.8$ \\
Height $(\mathrm{cm})$ & $169.9 \pm 6.5$ \\
Weight $(\mathrm{kg})$ & $60.5 \pm 10.4$ \\
Body fat $(\%)$ & $12.8 \pm 2.6$ \\
BMI $\left(\mathrm{kg} / \mathrm{m}^{2}\right)$ & $20.8 \pm 2.3$ \\
$\mathrm{LBM}(\mathrm{kg})$ & $52.6 \pm 7.9$ \\
$\mathrm{VO}_{2 \text { peak }}(\mathrm{ml} / \mathrm{kg} / \mathrm{min})$ & $42.0 \pm 2.8$ \\
\hline
\end{tabular}

Values are mean $\pm \mathrm{SD}$.

BMI: body mass index; LBM: lean body

mass; $\mathrm{VO}_{2 \text { peak }}$ : peak oxygen uptake.

Table 2. Changes in $\mathrm{VO}_{2 \text { peak }}$ and RPE during aerobic exercise

\begin{tabular}{lcc}
\hline & 15 min (First try) & 15 min (Second try) \\
\hline $\mathrm{VO}_{2 \text { peak }}(\%)$ & $63.0 \pm 2.2$ & $64.0 \pm 1.3$ \\
Final RPE & $12.3 \pm 2.1$ & $12.3 \pm 1.7$ \\
\hline
\end{tabular}

Values are mean $\pm \mathrm{SD}$.

$\mathrm{VO}_{2 \text { peak }}$ : peak oxygen uptake; RPE: rating of perceived exertion.

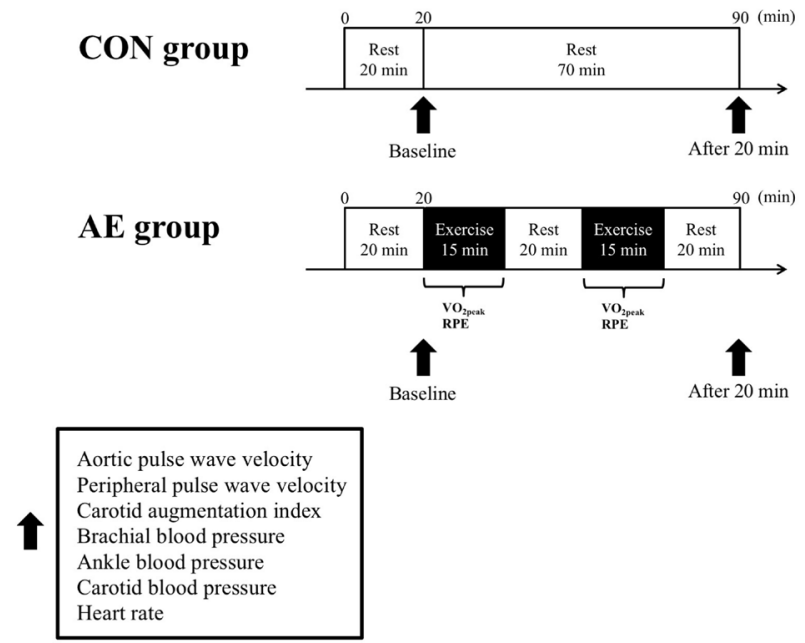

Fig. 1. Study design.

After 20 min of supine rest, aortic and peripheral pulse wave velocity, carotid augmentation index, brachial and ankle BP, HR were measured before (baseline) and at $20 \mathrm{~min}$ for the both groups. BP: blood pressure; HR: heart rate.

The ethics committee at Nippon Sport Science University approved this study, which proceeded in accordance with the guidelines for human experimentation published by our institutional review board (016-H068). This study also conformed to the principles of the Declaration of Helsinki. Each subject participated in two groups in crossover fashion: (1) accumulated aerobic exercise (AE group); and (2) the "CON group", which performed no accumulated aerobic exercise. Instead, the participant remained seated rest in a quiet room. After $20 \mathrm{~min}$ of supine rest, each subject performed a cycling exercise in two 15 min intervals (separated by 20 min rest) at $65 \% \mathrm{VO}_{2 \text { peak }}$, using a cycle ergometer. Oxygen uptake and carbon dioxide production were monitored breath-by-breath using an AE100i (Minato Medical Science Co., Ltd., Osaka, Japan) monitor. The carotid-femoral PWV (cfPWV), femoral-ankle PWV (faPWV), carotid augmentation index (AIx), brachial, ankle and carotid blood pressure (BP), and heart rate (HR) were measured using a PWV/ABI vascular testing device ${ }^{9)}$ (Omron-Colin Co., Ltd., Kyoto, Japan), both before (baseline), and $20 \mathrm{~min}$ after exercise (Fig. 1). The daily coefficients of variation (CVs) in the laboratory were $3 \pm 1 \%, 3 \pm 2 \%$ and $6 \pm 1 \%$ for cfPWV, faPWV and carotid AIx, respectively. The subjects abstained from alcohol, caffeine consumption, and exercise for $24 \mathrm{~h}$ before being tested. Each subject recovered for 7 days following each group. They reported to the laboratory after $12 \mathrm{~h}$ of fasting. $\mathrm{VO}_{2 \text { peak }}$ (highest value during exercise) was assessed using an incremental test to exhaustion (warm-up 2 min at $0 \mathrm{~W}$, cycling at $50 \mathrm{~W}$, followed by a $30 \mathrm{~W} / 2$ min increase) $)^{5)}$ one week before starting the study. Oxygen uptake and carbon dioxide production were monitored breath-by-breath using an AE300S monitor (Minato Medical Science Co., Ltd., Osaka, Japan). HR was determined during the exercise (BSM-2401, Nihon Kohden Co., Ltd., Tokyo, Japan). Rating of perceived exertion (RPE) was collected at the end of each three-min stage ${ }^{10)}$. All data are presented as means \pm standard deviation (SD). Means from the two intervals were compared using paired t-tests. Data were analyzed using repeated-measures 2-way ANOVA (group-by-time). Significant differences between mean values were identified using the Bonferroni post hoc test. Data were statistically analyzed using SPSS (version. 22; IBM, Armonk, NY, USA). Statistical significance was set at $\mathrm{p}<0.05$.

\section{RESULTS}

The $\mathrm{VO}_{2 \text { peak }}$ and final RPE during aerobic exercise did not differ between the first and second exercise period ( $\mathrm{p}>0.05$, Table 2). The cfPWV, faPWV and carotid AIx did not differ between groups ( $>00.05)$. These values decreased from baseline at 20 min for the AE group ( $\mathrm{p}<0.05)$, but for the CON group ( $>0.05$, Table 3 ). The brachial BP, ankle BP, carotid SBP, and HR did not differ between groups ( $>0.05$, Table 4$)$. The brachial BP, ankle BP, and carotid SBP did not change between baseline at $20 \mathrm{~min}$ for both groups ( $>>0.05$, Table 4). HR increased from baseline at $20 \mathrm{~min}$ for the AE group ( $<<0.05)$, but not for the CON group ( $>0.05$, Table 4$)$.

\section{DISCUSSION}

The novel finding of this study is that aortic and peripheral PWV decreased from baseline at 20 min for the AE group, but 
Table 3. Changes in arterial stiffness before and after exercise

\begin{tabular}{lll}
\hline & Baseline & After 20 min \\
\hline cfPWV $(\mathrm{cm} / \mathrm{sec})$ & & \\
AE group & $876.0 \pm 141.0$ & $762.4 \pm 155.6^{*}$ \\
CON group & $838.8 \pm 96.3$ & $832.9 \pm 105.7$ \\
faPWV $(\mathrm{cm} / \mathrm{sec})$ & & \\
AE group & $956.6 \pm 87.9$ & $859.7 \pm 68.4^{*}$ \\
CON group & $917.7 \pm 88.6$ & $922.1 \pm 89.6$ \\
Carotid AIx $(\%)$ & & \\
AE group & $-18.9 \pm 9.5$ & $-32.7 \pm 9.1^{*}$ \\
CON group & $-23.3 \pm 10.6$ & $-19.2 \pm 12.9$ \\
\hline
\end{tabular}

Values are mean \pm SD.

cfPWV: carotid-femoral pulse wave velocity; faPWV: femoral-ankle pulse wave velocity; AIx: augmentation index; AE group: accumulated aerobic exercise group; CON group: non-exercise control group.

$* \mathrm{p}<0.05$ vs. baseline.
Table 4. Changes in cardiovascular indices before and after exercise

\begin{tabular}{lcc}
\hline & Baseline & After 20 min \\
\hline Brachial SBP (mmHg) & & \\
AE group & $108.9 \pm 9.3$ & $108.8 \pm 10.0$ \\
CON group & $108.8 \pm 13.6$ & $110.7 \pm 11.6$ \\
Brachial DBP (mmHg) & & \\
$\quad$ AE group & $64.8 \pm 5.5$ & $64.1 \pm 6.6$ \\
CON group & $64.1 \pm 8.4$ & $64.4 \pm 7.6$ \\
Brachial PP (mmHg) & & \\
AE group & $44.1 \pm 6.0$ & $44.7 \pm 5.4$ \\
CON group & $44.7 \pm 7.7$ & $46.2 \pm 6.6$ \\
Ankle SBP (mmHg) & & \\
AE group & $126.8 \pm 11.7$ & $122.0 \pm 9.4$ \\
CON group & $124.6 \pm 13.3$ & $126.1 \pm 12.3$ \\
Ankle DBP (mmHg) & & \\
AE group & $67.2 \pm 5.7$ & $65.1 \pm 8.4$ \\
CON group & $65.8 \pm 9.5$ & $68.9 \pm 7.7$ \\
Ankle PP (mmHg) & & \\
AE group & $59.6 \pm 7.8$ & $56.9 \pm 6.6$ \\
CON group & $58.8 \pm 10.6$ & $57.2 \pm 7.6$ \\
Carotid SBP (mmHg) & & \\
AE group & $118.2 \pm 13.1$ & $116.1 \pm 12.3$ \\
CON group & $117.4 \pm 10.2$ & $116.5 \pm 10.5$ \\
Heart rate (beats/min) & & \\
AE group & $55.4 \pm 5.9$ & $64.1 \pm 6.0^{*}$ \\
CON group & $56.3 \pm 7.2$ & $53.6 \pm 7.0$ \\
\hline
\end{tabular}

Values are mean \pm SD.

SBP: systolic blood pressure; DBP: diastolic blood pressure; PP: pulse pressure; AE group: accumulated aerobic exercise group; CON group: non-exercise control group.

$* p<0.05$ vs. baseline.

not for the CON group. This suggests that acute accumulated aerobic exercise decreases aortic and peripheral PWV.

Individuals should pursue moderate-intensity aerobic physical activity to maintain and improve health. Moderate-intensity aerobic physical activity can be accumulated toward the 30 min minimum by performing 10 min interval training ${ }^{11)}$. Kingwell et al. ${ }^{4)}$ reported that moderate-intensity (65\% maximal oxygen uptake) acute aerobic exercise for 30 min decreases aortic and peripheral PWV at 30 minutes in healthy young males. Zheng et al. ${ }^{8}$ found that accumulated aerobic exercise (two 15 min intervals of cycling, performed 20 min apart) gave better effects with respect CAVI compared with control and one continuous 30 min interval. In the present study, aortic and peripheral PWV decreased from baseline at 20 min in the AE group, but not in the CON group. Thus, accumulated aerobic exercise might be effective at decreasing aortic and peripheral PWV. Decreased cfPWV and faPWV in the AE group might have been similar magnitude of the response to continuous aerobic exercise. We previously reported that cfPWV and faPWV decreased by up to 65 and up to $58 \mathrm{~cm} / \mathrm{sec}$ at $20-30 \mathrm{~min}$ after continuous aerobic exercise ( $30 \mathrm{~min}, 65 \% \mathrm{VO}_{2 \text { peak }}$ ) in untrained healthy young men ${ }^{12)}$, a similar effect to what we observed in the AE group. We believe this study might be more applicable to individuals who participate in low-level physical activity. One limitation of our study is that we did not compare accumulated aerobic exercise and continuous aerobic exercise.

We found that HR significantly increased from baseline at 20 min in the AE group, but not in the CON group. It has been reported that acute moderate-intensity aerobic exercise for $30 \mathrm{~min}$ decreases aortic and peripheral PWV, but increases HR at 30 min thereafter in healthy young men ${ }^{4)}$. PWV is unaffected by HR at frequencies $<120$ beats $/ \mathrm{min}^{13)}$. Thus, increases in HR after acute aerobic exercise might have a smaller effect on increases in PWV.

In addition to the aforementioned, there are other limitations to address. Our participants were healthy young males, which precludes generalizing our findings to middle-aged, elderly or female individuals. The sample size was small $(\mathrm{n}=9)$, although the magnitude of the effect on PWV was adequate and similar to those in previous studies of acute aerobic exercise on $\left.\mathrm{PWV}^{4}, 14,15\right)$. We did not design this study to examine the possible mechanisms by which accumulated aerobic exercise decreases aortic and peripheral PWV. Finally, we did not compare accumulated aerobic exercise and continuous aerobic exercise. Thus, the decrease in PWV reported in this study may be influenced not by cumulative exercise, but by independent exercise (continuous exercise) itself.

In conclusion, aortic and peripheral PWV decreased at 20 minutes in the AE group. This suggests that acute accumulated aerobic exercise decreases aortic and peripheral PWV. 
None.

\section{ACKNOWLEDGEMENT}

We thank Robert Lindeman, MD, PhD, from Edanz Group (www.edanzediting.com/ac) for editing a draft of this manuscript.

\section{REFERENCES}

1) Yamashina A, Tomiyama H, Arai T, et al.: Nomogram of the relation of brachial-ankle pulse wave velocity with blood pressure. Hypertens Res, 2003, 26: 801-806. [Medline] [CrossRef]

2) van Sloten TT, Schram MT, van den Hurk K, et al.: Local stiffness of the carotid and femoral artery is associated with incident cardiovascular events and allcause mortality: the Hoorn study. J Am Coll Cardiol, 2014, 63: 1739-1747. [Medline] [CrossRef]

3) Tsuchikura S, Shoji T, Kimoto E, et al.: Central versus peripheral arterial stiffness in association with coronary, cerebral and peripheral arterial disease. Atherosclerosis, 2010, 211: 480-485. [Medline] [CrossRef]

4) Kingwell BA, Berry KL, Cameron JD, et al.: Arterial compliance increases after moderate-intensity cycling. Am J Physiol, 1997, 273: H2186-H2191. [Medline]

5) Heffernan KS, Collier SR, Kelly EE, et al.: Arterial stiffness and baroreflex sensitivity following bouts of aerobic and resistance exercise. Int J Sports Med, 2007, 28: 197-203. [Medline] [CrossRef]

6) Wang H, Zhang T, Zhu W, et al.: Acute effects of continuous and interval low-intensity exercise on arterial stiffness in healthy young men. Eur J Appl Physiol, 2014, 114: 1385-1392. [Medline] [CrossRef]

7) Zhou Z, He Z, Yuan M, et al.: Longer rest intervals do not attenuate the superior effects of accumulated exercise on arterial stiffness. Eur J Appl Physiol, 2015, 115: 2149-2157. [Medline] [CrossRef]

8) Zheng L, Zhang X, Zhu W, et al.: Acute effects of moderate-intensity continuous and accumulated exercise on arterial stiffness in healthy young men. Eur J Appl Physiol, 2015, 115: 177-185. [Medline] [CrossRef]

9) Okamoto T, Sakamaki MS, Min SK, et al.: Repeated cessation and resumption of resistance training attenuates increases in arterial stiffness. Int J Sports Med, 2015, 36: 440-445. [Medline] [CrossRef]

10) Borg GA: Perceived exertion: a note on "history" and methods. Med Sci Sports, 1973, 5: 90-93. [Medline]

11) Haskell WL, Lee IM, Pate RR, et al. American College of Sports Medicine American Heart Association: Physical activity and public health: updated recommendation for adults from the American College of Sports Medicine and the American Heart Association. Circulation, 2007, 116: 1081-1093. [Medline] [CrossRef]

12) Kobayashi R, Hatakeyama H, Hashimoto Y, et al.: Acute effects of different aerobic exercise duration on pulse wave velocity in healthy young men. J Sports Med Phys Fitness, 2017, 57: 1695-1701. [Medline]

13) Callaghan FJ, Babbs CF, Bourland JD, et al.: The relationship between arterial pulse-wave velocity and pulse frequency at different pressures. J Med Eng Technol, 1984, 8: 15-18. [Medline] [CrossRef]

14) Maeda S, Tanabe T, Otsuki T, et al.: Acute exercise increases systemic arterial compliance after 6-month exercise training in older women. Hypertens Res, 2008, 31: 377-381. [Medline] [CrossRef]

15) Sugawara J, Maeda S, Otsuki T, et al.: Effects of nitric oxide synthase inhibitor on decrease in peripheral arterial stiffness with acute low-intensity aerobic exercise. Am J Physiol Heart Circ Physiol, 2004, 287: H2666-H2669. [Medline] [CrossRef] 